\title{
MECANISMOS DE INFLUENCIA EN EL CONSUMIDOR INFANTIL DE VILLAVICENCIO ${ }^{1}$
}

\author{
Laura Catalina Gallego Esquivel ${ }^{2}$ \\ Yehimi Carolina Higuera ${ }^{3^{*}}$ \\ María Cristina Otero Gómez ${ }^{4}$ \\ Wilson Giraldo Pérez ${ }^{5}$
}

Para citar este artículo: Gallego, L., Higuera, Y., Otero, M. y Giraldo, W. (2014).

"Mecanismos de influencia en el consumidor infantil de Villavicencio". Inquietud Empresarial. Vol. XIV (2), p.p 117-130

Fecha de Recepción: 25 de junio de 2014

Fecha de Aceptación: 3 de septiembre de 2014

\footnotetext{
1 Artículo de investigación científica elaborado por el grupo de Investigación Dinámicas de Consumo de la Universidad de los Llanos, entidad financiadora del proyecto de investigación "El consumo infantil en el nivel socioeconómico medio de Villavicencio Colombia".

2 Profesional en Mercadeo. Universidad de los llanos. laura.gallego@unilanos.edu.co

3 Profesional en Mercadeo. Universidad de los Llanos. yehimi@hotmail.es

4 Magister en Mercadeo. Docente Universidad de los Llanos. motero@unillanos.edu.co

5 Magister en Mercadeo. Docente Universidad de los Llanos. wgiraldo@unillanos.edu.co
}

Volumen XIV (2) (Julio - Diciembre 2014). ISSN 0121-1048 


\title{
RESUMEN
}

El presente artículo expone parte de los resultados de la investigación titulada“El consumo infantil del nivel socioeconómico medio de Villavicencio (Colombia)". El objetivo consistió en identificar los mecanismos de influencia en la formación de preferencias de consumo de los niños entre 5 y 9 años de edad. Los hallazgos permitieron demostrar la existencia de varios agentes que inducen en la realización del intercambio comercial como son: la familia y los adultos, los semejantes, la publicidad y la compra icónica. Adicionalmente se analiza el grado de influencia que representa la tecnología en este grupo etario. Dichos agentes han contribuido en la redefinición de los perfiles de este segmento, caracterizados por estar cada vez más informados y con autonomía para tomar decisiones a la hora de adquirir productos o de influir en las compras de quienes conforman su núcleo familiar.

\section{PALABRAS CLAVE}

Influencia, consumo, niños, publicidad, mercadeo.

\begin{abstract}
This paper presents part of the results of the research entitled "The children's consumption of middle socioeconomic level Villavicencio (Colombia)". The aim was to identify the mechanisms of influence on consumer preference formation of children between 5 and 9 years old. The findings allow to establish the existence of various inducing agents in the conduct of trade as are the family and adults, alike, iconic advertising and shopping. Additionally, the degree of influence that represents technology in this age group is analyzed. Such agents have contributed to the redefinition of the profiles in this segment, characterized by being increasingly informed and autonomy to make decisions when purchasing products or to influence the purchases of those who make your househol
\end{abstract}

\section{KEYWORDS}

Consumption, children, influence, advertising, marketing.

\section{INTRODUCCIÓN}

Actualmente el Mercadeo como disciplina y como área organizacional de las empresas, debe replantear la forma de analizar el consumo en los diferentes segmentos. Esto se debe a que se han presentado cambios que marcan nuevas pautas entre las relaciones de oferta y demanda, principalmente si se comparan con los postulados correspondientes a épocas anteriores. Por ejemplo, Taylor a finales del siglo XIX basaba esta relación otorgándole total importancia a la producción de bienes, dejando de lado el análisis de las necesidades de la demanda. Así mismo, Ford en el siglo XX aunque avanzó hacia la expansión interclasista del mercado, no implementó estrategias orientadas al estudio de las necesidades de los consumidores. Tanto Taylor como Ford hicieron grandes contribuciones a los modelos de producción, pero se identifican con la Ley de Say de inicios del siglo XIX donde cada oferta crea su propia demanda. 
En la actualidad, aunque se mantienen vigentes algunas de las tesis planteadas por estos teóricos, surgen cambios a nivel social, cultural, político y económico. Los cuales traen consigo el surgimiento de nuevas relaciones al interior de las familias, por ejemplo, el involucramiento de sus miembros del núcleo familiar en las decisiones de consumo. Para el caso de los niños, estos cobran protagonismo al considerarse que tienen la posibilidad de influir en la decisión de compra de bienes y servicios.

Un informe presentado por Sylvia Carazo (2009), Gerente de Mercadeo de la empresa CRG TOYS de Costa Rica, revela algunas características del segmento infantil y la importancia que representa este mercado para muchas empresas. El estudio presenta dos situaciones, por un lado están los factores que influyen en el niño a la hora de tomar decisiones de compra, por el otro, revela las diferentes posturas del menor como influenciador del proceso de compra. Dentro de los factores que influyen la compra, se tiene que los niños poseen más retentiva, esto les permite asociar un anuncio televisivo con algún tipo de material publicitario exhibido en el punto de venta. Otros elementos influenciadores se apoyan en los diferentes modelos a seguir, es decir basados en la imitación, tales como personajes reales representados en los compañeros de colegio y los hermanos mayores. Sumados a estos, también se encuentran los personajes ficticios, quienes cobran importancia, toda vez que sus características querrán imitarse para sentirse fuerte, veloz, valiente, como también bonita, simpática, etc.

Como puede apreciarse, la identidad de género también juega un papel importante en cuanto al modelo que se quiera seguir o imitar, llegando a recibir críticas sobre la influencia que tienen los comics o dibujos animados en el consumo infantil. Por ejemplo, son las niñas las que poseen más juguetes de este tipo, generalmente de manufactura Disney, mientras que los niños apuestan más por juguetes agresivos inspirados en las series niponas, contribuyendo a subrayar una marcada influencia sexista en la aceptación de juguetes para unas y para otros (Pérez, 1999). El mismo informe expone que los infantes quieren tener todo y antes que los demás, lo que representa que el niño cambie su rol, pasando de influenciado a influenciador en las decisiones familiares de consumo, tanto en padres como en abuelos y familiares cercanos.

Otro estudio efectuado en la ciudad de Cali - Colombia, dirigido a niños de 6 a 9 años de edad, permitió concluir que estos consumidores establecen relaciones fuertes con los productos en el sentido de que se identifican con ellos por aspectos emocionales trabajados por las diferentes marcas (Portela, 2011). Al respecto, cita el ejemplo de las bebidas instantáneas de chocolate que para ellos su consumo representa mayor nutrición, fuerza y crecimiento. De igual forma las mascotas publicitarias también los animan a inclinarse por el producto que representan. No obstante, la investigación expresa que las relaciones no son perdurables, debido al dinamismo que presenta el mercado en cuanto a gustos y estilos que son fácilmente influenciables por las tendencias en el entorno. Adicionalmente, revela que los niños aunque son receptivos, también son críticos de la forma como se les transmite el mensaje, de allí que las agencias publicitarias trabajen de manera incesante para atraerlos y retenerlos con estrategias donde el diseño, las texturas y el color les permitan alcanzar la aceptación de este segmento.

Aunque se trata de un grupo bastante atractivo para desarrollar estrategias de comunicación, también requiere de un cuidado especial para evitar hacer el tránsito del consumo al consumismo. Asumiendo una posición de cuestionamiento, Álvarez (2008), en un estudio desarrollado en España para determinar los efectos de la 
publicidad y los medios de comunicación en infantes, manifiesta que la importancia de los niños como público para las campañas de publicidad y para el medio televisivo ha cambiado notablemente. Anteriormente no eran considerados como un potencial que les podría generar rentabilidad, razón por la cual la franja de comerciales era indiferenciada pues los niños eran considerados como un número más en la audiencia. En la actualidad, existen dos factores que no dejan de crecer y que parecen estar lejos de llegar a su límite. Se trata en primer lugar de la renta disponible de los niños para efectuar sus compras personalmente. En segundo lugar, el autor español le atribuye a la capacidad de influencia que el niño ostenta sobre las decisiones de una parte importante del gasto familiar.

Otros elementos que influencian las preferencias en consumo son los citados por Sedeño (2005), quien argumenta que existen dos efectos de orden global que pueden homogenizar el consumo. Así pues, el primero hace referencia a la tendencia creciente del niño a imitar el mundo adulto y el segundo, está orientado a la uniformización de los gustos infantiles a escala mundial teniendo en cuenta que las necesidades, estilos de vida y valores emergentes permiten el uso de una publicidad más uniforme.

Afirma Schor (2004) que los niños entre 6 y 12 años y los adolescentes conforman hoy por hoy la generación más preocupada por las marcas y el consumo a todo lo largo de la historia; por ello es importante destacar la influencia que tienen en los infantes, los semejantes en su rol de estudiantes, y los diferentes medios de comunicación. Este investigador organiza los mecanismos de influencia en cuatro grupos a saber. El primero corresponde a los semejantes, entendido este mecanismo como la capacidad que tiene un igual para modificar mi propia conducta. En segundo lugar se encuentra la comunicación, que es el mecanismo que utilizan las empresas para masificar los conocimientos acerca de su producto. El siguiente grupo de influenciadores están relacionados con los temas icónicos del consumo de determinado bien o servicio. Finalmente, los adultos son considerados influenciadores cercanos, toda vez que el infante confía en el criterio y/o buen juicio de sus cuidadores.

Continuando con esta línea, para Breé (1995) el consumo de los niños se ve influenciado por tres factores debidamente identificados uno son los iguales, aquellas personas que los niños perciben como sus semejantes y quienes juzgan y dan las aprobaciones si se pertenece a un grupo en especial que es de interés para el niño, por otro lado están las escuelas cuyo papel en el consumo infantil es el de reforzar las experiencias de consumo y de enseñar sobre el mismo; por último está la publicidad y los medios de comunicación que crean en los niños expectativas y estereotipos de consumo.

Como puede apreciarse, son múltiples los elementos y análisis que plantean diversos autores y estudios que demuestran la importancia de estudiar con más detalle al consumidor infantil. Para el caso de Villavicencio, se encontraron algunos elementos afines a los ya estudiados en otros contextos, tanto nacionales como internacionales, los cuales se darán a conocer con más detalle en el presente escrito

\section{METODOLOGÍA}

Las características de la investigación motivan a la utilización del paradigma relativista como base epistemológica, el cual busca conocer e interpretar el comportamiento de los seres humanos desde una perspectiva más integral que descriptiva, en ella la conducta de cada persona es analizada de una manera verdaderamente 
contextualizada y en función de los componentes subyacentes; adicionalmente busca explicaciones de los participantes de cualquier tipo de intercambio con miras a obtener la satisfacción integral, implicado en la concepción e implementación del marketing.

De esta forma y partiendo del sustento relativista la investigación que se desarrolló es de orden cualitativa. Para el desarrollo investigativo, este trabajo asumió realidades propuestas por Creswell (1994) quien plantea que el investigador actúa desde lo epistémico con el objeto de investigación; desde lo ontológico con una realidad subjetiva; desde lo axiológico con la realidad influencia por valores de cada sujeto; desde lo retórico se basa en el lenguaje no verbal, y desde la perspectiva metodológica por el proceso inductivo, donde su diseño es emergente. Las categorías se identifican durante el proceso investigativo, y la veracidad y confiabilidad se hacen a través de la verificación y no de la validez interna.

Bajo la anterior perspectiva, la propuesta de investigación se dirigió de manera premeditada a optar por un contexto de descubrimiento. Así, se trató de inducir y no de deducir, de comprender y no de explicar. Teniendo en cuenta que desde la etnografía el investigador es prácticamente el instrumento para recopilar información, se hizo una preparación previa que permitió acercarse a la realidad de este grupo etario de una forma más clara.

Las técnicas implementadas en el presente estudio con el fin de recopilar información se basaron en grupos focales, y observación no participante. En los grupos focales se realizaron sesiones donde participaron en un bloque niños y niñas de 5-7 años y en el otro niños y niñas de 8-9 años en las cuales se hicieron grabaciones de audio y video con el fin de realizar la transcripción respectiva, y al final de cada una de ellas se realizó un proceso de compra simulada donde cada sujeto era observado por los investigadores sin interactuar con él.

Para el análisis e interpretación de los datos recopilados: se realizó y transcribió la primera sesión de grupo, sobre la cual se hizo un análisis piloto. Este análisis permitió corregir las preguntas en función de orientarlas de una manera más apropiada hacia los objetivos de la investigación, aunque la información que aportó fue igualmente importante e incluida. Se elaboró un resumen de las preguntas y respuestas de los participantes, y se analizó la información de manera horizontal, con lo cual se formó un concepto general y una primera comprensión del fenómeno global, así mismo se detectaron los desacuerdos significativos entre los informantes en cuestiones específicas.

Una vez realizado este proceso, se identificaron los mecanismos de influencia en el comportamiento del consumidor infantil en edades comprendidas entre 5 y 9 años de Villavicencio (Colombia).

\section{RESULTADOS}

Esta sección está dividida en dos partes, la primera trata de los resultados obtenidos en el nivel socio económico bajo y la segunda expone los resultados obtenidos en el nivel socio económico medio, a su vez estos están subdivididos en los dos grupos de estudio niños de 5-7 años y niños de 8-9 años, para hacer del texto más entendible se contraponen los resultados obtenidos en cada grupo. 


\section{Influencia de la familia y adultos}

\subsection{Nivel socio económico bajo}

Como se mencionó anteriormente, como agentes influenciadores se encuentran los adultos, quienes a través de su orientación inciden en el consumidor infantil a la hora de tomar una decisión de compra. De acuerdo con la información obtenida, para el grupo de niños entre 5 -7 años se pudo identificar que tienen claridad sobre la importancia de los alimentos sanos y nutritivos. Su conocimiento se debe al evocar el momento en que sus padres realizan las compras de la canasta familiar y también como resultado de las enseñanzas en los colegios donde el profesor los persuade a aumentar el consumo de frutas.

Al respecto, Mondragón (2013) manifiesta que en el ciclo de vida familiar los niños reciben de sus padres los primeros estímulos sobre las compras. Bajo esta mirada, el entorno en el que se desenvuelven los niños debe ser considerado a la hora de realizar un estudio de mercados, toda vez que los hábitos de compra,los estilos de vida, los gustos y necesidades de la familia cambian paulatinamente en una dinámica de la que los niños forman parte importante.

A su vez Restrepo y Maya (2004), argumentan que el estado nutricional está determinado por factores económicos, sociales y culturales, donde la familia desempeña un papel destacado en la formación de los hábitos alimentarios en el escolar, de igual forma exponen que:

Las interacciones del niño con su medio ambiente, y en especial con el encargado de su cuidado - la madre o quien hace sus veces-, cobran particular importancia en los primeros años de vida. Las características de este vínculo afectivo pueden favorecer o afectar negativamente en la formación de gustos y rechazos (Restrepo y Maya, 2004).

En los niños más grandes, es decir quienes están en el rango de 7 a 9 años de edad, la característica afín se basa en la adquisición de cierta autonomía para pedir lo que desean. Estos infantes expresan libremente sus deseos de compra, de tal manera que se convierten en decisores de las marcas incluidas en el desayuno.

No obstante, según Macías y otros (2012) argumentan que los padres deben decidir la cantidad y calidad de los alimentos proporcionados y junto con ellos está el acompañamiento de los profesores quienes juegan un rol fundamental en el fomento y adquisición de hábitos alimentarios a través de la promoción y educación para la salud.

Con estos resultados queda evidenciado que los niños han ganado un espacio que en épocas anteriores estaba destinado exclusivamente a los padres o adultos. Hacia la década de los años 70 y 80 en Colombia, principalmente en las poblaciones pequeñas existían compras de bienes y servicios que aunque el consumidor final era el niño, éste no tenía la capacidad de decidir su adquisición, tal era el caso del vestuario, algunas actividades de ocio y principalmente los alimentos. En la actualidad el panorama es otro, pues los niños ejercen gran influencia sobre los productos que se compran, especialmente en los alimentos.

\subsection{Nivel socio económico medio}

Al igual que el segmento anterior, la orientación que brinda la familia influye en la 
construcción del perfil del consumidor infantil. Sin embargo se presentan algunas diferencias, por ejemplo, en el caso de adquirir los alimentos que se incluyen en las loncheras, los niños de 5 a 7 años afirman que la decisión de compra recae en los padres; no sucede lo mismo en el caso de las niñas de la misma edad pese a que son los padres quienes realizan la transacción, son ellas quienes deciden que incluir en la compra. En el caso de los rangos de 8 a 9 años de edad, tanto niñas como niños deciden qué comprar, pero los padres escogen el lugar de compra que generalmente corresponde a los almacenes de cadena. En la mayoría de casos los niños acompañan a los padres a realizar esta actividad y es percibida por los infantes como una actividad familiar.

Al respecto, Ruiz (1996) describe el papel de los padres dentro del proceso de formación del consumidor y lo divide en tres momentos, el primero aprendizaje por observación cuando va a mercar con la familia; el segundo es por la participación cuando le dejan decidir que va a llevar para su lonchera; y el tercero por entrenamiento cuando le obliga a hacer cálculos. Sobre esta afirmación y aplicado al caso de Villavicencio, se toman en cuenta los dos primeros, pues en el tercer caso los niños no administran el dinero necesario para hacer las compras entendiéndose esta función como una responsabilidad de los padres.

\section{Influencia de lo semejantes}

Para identificar el grado de influencia de los semejantes, es decir consumidores infantiles de la misma edad, se adecuó un espacio con todas las características de un mercado donde existían 4 tiendas. A cada niño se le entregó COP50.000 para que realizaran compras, teniendo como opciones dentro de la oferta disponible establecimientos especializados en artículos infantiles de aseo y tocador, dulces, alimentos saludables y snacks.

Durante el proceso, se pudo evidenciar que en ambos segmentos el liderazgo fue asumido por el género masculino, pues fueron ellos quienes intervinieron y tomaron la iniciativa para empezar el proceso de exploración frente a las diferentes opciones que presentaba el mercado. Sin embargo, una vez familiarizados con el entorno, tanto niñas como niños decidieron comprar de forma independiente.

\subsection{Nivel socioeconómico bajo}

De acuerdo con los resultados y observaciones obtenidas, en el grupo de niños entre los 5 y 7 años de edad se presentó de manera frecuente que los de menor edad en oportunidades buscaran a los niños que estaban en el límite de los 7 años, lo cual les generaba seguridad en el momento de realizar las compras. Para el caso de las niñas, las que tenían aproximadamente 5 años siguieron a la líder de mayor edad para ingresar a la tienda de artículos infantiles de aseo y tocador.

Entre tanto, en el grupo de los niños pertenecientes al rango de 8y 9 años, el niño de mayor edad fue seguido por los demás integrantes sin presentar diferencias de género.

Aunque las personas tienen diferentes razones para comprar, podría afirmarse que este hecho involucra motivaciones inspiradas en las necesidades básicas insatisfechas, las fuerzas psicológicas y las presiones sociales. Al menos así lo reveló este ejercicio. Por ejemplo, algunos niños una vez entrados en confianza con la locación y abandonada la presión del líder, se inclinaron de forma desmesurada hacia la adquisición de artículos de aseo, incluyendo al niño que en su momento asumió el liderazgo. Se pudo apreciar que las primeras compras fueron crema 
dental, cepillos de dientes, shampoo, etc que en su mayoría estaban envasados en figuras alusivas a las princesas de Disney y los superhéroes de Marvel. La razón de esta decisión, aunque en principio parecía que estaban motivados por los comics, tuvo otros argumentos. Al preguntarles por qué se inclinaron por estos productos como primera opción de compra, la respuesta generalizada fue porque en sus casas no tenían la posibilidad de acceder a ellos, pues las compras se hacen en envases de tamaño familiar y con la marca que deciden los papás. En este caso la marca no fue relevante, pues también adquirieron productos que no tenían un diseño infantil atractivo, pero que de alguna manera podrían utilizarlo de manera individual, pues el contenido estaba diseñado exclusivamente para niños evitando el riesgo de que en sus casas lo adultos hicieran uso de ellos.

En cuanto a la presión social y de manera independiente al nivel socioeconómico al que pertenezcan, los niños objeto de estudio quisieron tener lo mismo que poseían los demás y en lo posible adelantarse a adquirirlo.

En síntesis, existió la presencia de unos líderes en el momento de explorar el mercado, pero una vez entraron en confianza todos se inclinaron por la tienda de aseo infantil, de tal manera que agotaron todas las existencias del establecimiento, dando lugar a la búsqueda de otras opciones de compra.

En segundo lugar en orden de preferencias, los niños ingresaron a las tiendas de dulces y snacks. Se pudo establecer que algunas marcas tradicionales en Colombia dentro de la categoría de golosinas como es bombombum, que otrora fuera considerada uno de los productos más apetecidos por el público infantil, pasó desapercibido en el proceso de compra, pasando a ser reemplazado por otros dulces de menor precio. Empero, dentro del abanico de posibilidades que encontraban los niños, algunos prefirieron inclinarse por productos que nunca habían consumido y que incluyeron en su cesta de compras como resultado del rol influenciador que ejercían los hermanos en casa.

Al respecto, se presentó el caso de una niña de 8 años quien nunca había probado Choco Popsel cual está en la categoría de cereales, pero debido a que su hermano mayor lo consume, ésta quiso emular su comportamiento para confirmar o contradecir el concepto que tenía el consumidor inicial. Esta reacción es explicada por Núñez (2013) quien afirma lo siguiente:

En relación a los hábitos, los hermanos tienen mayor impacto en la vida de sus hermanos que los mismos padres. La influencia es superior ya sea que se refieran a hábitos positivos o negativos, hay más probabilidades que los hermanos menores imiten a los mayores que a sus padres".

\subsection{Nivel socioeconómico medio}

En este segmento, el liderazgo presentado al interior del grupo se manifestó en un primer momento principalmente en los hombres quienes ejercían de forma espontánea ese papel, mientras las niñas así lo aceptaban. Aunque aparentemente se evidencia un comportamiento sexista, es importante abordarlo con una mirada que trasciende la cuestionada disputa de poder entre hombres y mujeres, donde ampliar esta interpretación se remite a concepciones antropológicas. Según los explica Sanday (1981) las mujeres delegaron el liderazgo a los hombres, quienes siempre se han mostrado fuertes y han basado su liderazgo en la demostración de poder y fuerza, pero a su vez la autora hace un paréntesis e indica que las mujeres 
no se alejan del poder y que siguen siendo importantes e influyentes en la toma de decisiones.

Ahora bien, desde una perspectiva económica, Ruiz (1996) citando a Breé establece que los grupos y los líderes son para los niños una fuente de seguridad y de autonomía a la hora de adquirir un producto. De este modo, tanto niños como niñas buscan emular o imitar a un líder, independiente del género al que pertenezca, así lo expresan Lázaro y Mayoral (2005) al indicar que dentro de las fuentes de información que consultan los niños en el momento de adquirir un nuevo producto, el grupo social o grupos similares se encuentran en el segundo lugar.

De este modo, los rasgos más destacados en el nivel socioeconómico medio se basaron en seguir al líder a la tienda de golosinas. A diferencia del grupo anterior, los niños dejaron como última opción la tienda de implementos de aseo y tocador, pues consideraban que esos artículos estaban incluidos en las compras mensuales que efectuaban sus padres, por tanto su oferta no generó algún grado de novedad que motivara la compra como prioridad inmediata.

En la etapa intermedia del ejercicio de simulación de compra, los niños con pleno dominio del espacio compraron de manera tranquila, inclusive en algunos casos las niñas de mayor edad tomaron decisiones autónomas alejadas de quienes podrían ejercer algún tipo de influencia. En este sentido, las niñas se basaron más por la influencia de los padres, durante el proceso de comprar incluyeron productos saludables como frutas, agua y alimentos bajos en grasa. Estos últimos correspondían específicamente a las marcas de galletasTosh y Hony Bran, las cuales según los niños y confrontados con la información de los padres, forman parte de la demanda de alimentos incluida en la compra familiar.

\section{Influencia de la publicidad}

A menudo se suele afirmar que la publicidad genera efectos negativos en el niño quien los absorbe inmediatamente y comienza a evidenciar estas influencias en su conducta prácticamente al instante (Álvarez, 2008). Sin embargo, esta investigación arrojó algunos resultados que se alejan del anterior postulado. Previo al proceso de simulación de compra, los niños estuvieron expuestos a un reel de comerciales alusivos a las marcas y productos Kellogs, bombonbum, galletas festival, galletas tosh, yogurth Alpina, gelatina boggy, entre otros, no obstante en el momento de elegir las preferencias de compra no necesariamente contemplaban la totalidad de esos productos. De la tanda de comerciales se eliminó intencionalmente los productos asociados al aseo y belleza. Sin embargo tal como lo afirma Vygotski (1991) los niños toman decisiones según el entorno social y cultural al que pertenezcan, marcando algunas diferencias según el nivel socioeconómico.

\subsection{Nivel socioeconómico bajo}

Las compras realizadas por los niños de este nivel, tal como se mencionó anteriormente tuvo como prioridad la adquisición de elementos de aseo personal. En segundo lugar de preferencia, los pequeños optaron por la categoría de alimentos, especialmente de los paquetes individuales de cereales Kellogg's listos para el desayuno. En este segmento se pudo confrontar que pase a estar expuesto a comerciales que inducían al consumo de golosinas, primó la suplencia de necesidades básicas insatisfechas. Llegando al punto de que un niño tomó 7 unidades de cereales Kellogg's, cuándo se le preguntó sobre la razón de esa decisión, la respuesta estaba orientada al aprovisionamiento para los 7 días de la semana. Con esto se evidenció, por una parte el deseo de consumir productos nuevos que no son frecuentes en 
la canasta básica de su núcleo familiar, y por otro una preocupación por la salud. Ello es el resultado positivo de la influencia de la publicidad, puesto que los niños no se desbordan en compra de dulces, golosinas o productos no tan saludables, bajo esta perspectiva los avisos publicitarios en televisión constituyen una probable influencia sobre las elecciones alimentarias de los niños (Maryamy otros, 2005).

Las reacciones de los niños en el nivel socioeconómico bajo van en contravía de lo afirmado por Rojas (2008) quien plantea que en la infancia los comerciales tienen una gran influencia en promover el consumismo infantil y en generar en los padres presión por comprar determinadas marcas. Para el caso, cuando se tienen necesidades básicas insatisfechas y cuando los recursos financieros son limitados, es difícil dar espacio al consumismo tanto en padres como en niños.

\subsection{Nivel socio económico medio}

Otra afirmación realizada por Rojas (2008) consiste en argumentar que las campañas de publicidad han sufrido grandes cambios, esto se debe a que anteriormente el niño era considerado una parte más de la audiencia y no una de las más significativas desde el punto de vista de la rentabilidad. Por esta razón las firmas publicitarias destinan grandes esfuerzos tanto financieros como creativos para cautivar a este segmento. Sin embargo, en esta investigación se pudo comprobar que las respuestas varían dependiendo de la edad. En el caso de los niños de 5 a 7 años, la publicidad no causaba efectos de animación y encanto, caso contrario sucedió en los menores entre 8-9 años quienes observaban detenidamente los comerciales hasta llegar al punto de soltar risas cuando estos tenían contenidos humorísticos.

Investigadoras como Tur y Ramos (2008) reflexionan sobre el grado de disfrute de la publicidad, revelando que en la edad de los 7 a los 12 años este individuo puede recordar la publicidad que sea capaz de sincronizar la música, los anuncios dinámicos y los animales. Estas afirmaciones respaldan los resultados obtenidos en la investigación aplicada en Villavicencio.

Adicionalmente, las investigadoras afirman que los niños en edad pre-conceptual no establecen diferencias entre contenido televisivo y publicidad, esto causado por el desconocimiento de la finalidad de los comerciales, ocasionando una diferencia entre las actitudes y respuestas de los grupos frente al mismo estímulo.

Al realizar el paralelo entre marcas expuestas y compradas, el resultado para los niños entre 5 y 7 años y las niñas entre 5 y 9 años fue una compra efectiva de productos de la misma marca o de la misma categoría de la exposición publicitaria, lo que revela que los comerciales tuvieron en ellos el efecto deseado.

Es importante tener en cuenta que en el nivel socio económico medio se involucran más variables que no habían sido contempladas originalmente en la investigación como el nivel educativo, o la estabilidad laboral de los padres entre otras, pero que igualmente son importantes puesto que estas bloquean o facilitan la adquisición de determinadas categorías, productos y/o marcas. Demostrando así que pese al interés evidenciado por la mayoría de los niños en cuanto a observar los contenidos de los comerciales, algunos niños hicieron caso omiso al mensaje implícito que tenía determinada marca. Y aunque les pareció llamativo, se quedaron con las enseñanzas aprendidas en el seno de su familia, por ejemplo, una niña de 9 años que padece sobrepeso y que tiene propensión a la obesidad, dentro de sus compras incluyó frutas, galletas integrales y agua, argumentando que son las recomendaciones de sus padres y de la nutricionista que la orienta en los procesos alimenticios. 
En este sentido, la publicidad no ejerce una influencia absoluta sobre las decisiones de los niños y tampoco es totalmente negativa como se menciona en diferentes escritos. Por el contrario, se sobrepone la influencia que generan los padres, adultos y los semejantes en el momento de configurar un proceso de compra.

\section{Compra Icónica}

Algunos elementos correspondientes a esta tipología de compra están influenciados por las imágenes, símbolos, marcas, reconocimiento y estatus que algunos productos pueden brindar. En un estudio desarrollado por Del Moral (1999), sobre la compra de juguetes se pudo demostrar que un $60 \%$ de las compras de estos estaba influenciado por la aparición o representación de personajes de dibujos animados, considerando que todas las industrias conocen la puerta de entrada para llegar a la mente del pequeño consumidor.

En el caso de Villavicencio todos los niños y niñas entre 5 y 7 años independientemente del nivel socioeconómico hicieron compras en la categoría de aseo, agotando las existencias de la tienda, pese a que no se presentaron comerciales anunciando productos de esta categoría. Al respecto, se tiene que los niños al observar los productos con diseños ergonómicos y con figuras e imágenes de películas infantiles, causa en ellos gusto y atracción. Pero es importante aclarar que el producto por sí mismo en el nivel socioeconómico bajo no generó tal decisión, aunque sí influyó en la motivación de compra. A manera de complemento, el entorno social también hizo su aporte debido a que a los niños de este nivel no les compran productos destinados solo para ellos o con diseños atractivos e infantiles como crema dental pequeña con figuras infantiles, shampoo con formas ergonómicas, desodorante de princesas o superhéroes, gel o cepillos con imágenes, lociones escarchadas, etc. Algunas de las justificaciones de ellos respecto a las compras de dichos productos se basaban en la admiración por la imagen que tenía el producto, también porque los necesitaban, en el caso de crema dental decía una niña: "Porque mis papas tienen crema grande y yo quiero una chiquita para mí”.

En cuanto a los productos de la categoría de snacks y golosinas, los niños de 7 a 9 años demostraron un mayor grado de preferencia por estos últimos, no así las niñas quienes difícilmente se inclinaron por marcas como barrilete, goma de mascar tumix, entre otros.

De estas actitudes se desprende que está demostrado que la influencia de la publicidad icónica presenta resultados positivos en las ventas, por esta razón la decisión se centra en la elección adecuada del respectivo ícono.

\section{Influencia de la tecnología}

Los niños tienen la facilidad de adaptarse al alto grado de avance tecnológico que ha alcanzado el mundo. Para explicar este postulado Schor (2004) hace referencia a los niños postmodernos que nacieron en una era digital regida por las leyes del entretenimiento. Sin embargo, a la hora de consumir quedó demostrado que en infantes de edades preconceptuales la tecnología es vista como un elemento de entretención y uso del tiempo libre; y al aumentar su edad, la tecnología pasa a ser vista como una herramienta de trabajo de los adultos (Otero y Giraldo, 2014).

En el caso de los niños del nivel socioeconómico bajo, la exposición a la tecnología es mínima. Por un lado, en sus casas la tenencia de computadores se ve reducida y por otro, el acceso a internet es mínimo. De igual forma, se evidencia un desconocimiento sobre los pagos en línea y el uso de tarjetas débito y crédito así 
como la funcionalidad que estas representan para operaciones donde funciona el comercio electrónico.

En cuanto a los niños del nivel socioeconómico medio, sí conocen de la existencia de estas formas de pago y en todos los casos en sus hogares tienen acceso a computadores, tabletas y el uso de internet. Empero, sus padres no tienen como práctica de consumo realizar operaciones virtuales por temor al fraude, de tal forma que estas transacciones no son vistas en los pequeños como una forma de atender las necesidades presentadas en la demanda de bienes o servicios.

\section{CONCLUSIONES}

Los niños no todas las veces son conscientes de lo que consumen, pero sí poseen un alto grado de autonomía motivados por las creencias, valores y niveles de bienestar encontrados en su entorno. Además, los adultos principalmente los padres y en menor grado los profesores, son influenciadores del proceso de compra. Estos crean los primeros rasgos de consumo en el niño y a su vez estimulan las acciones que lo distinguirán como consumidor en el futuro.

De igual forma se cuentan como influenciadores los iguales o semejantes, quienes ejercen presión sobre las decisiones que los niños seguidores tomen. Esto se debe a que los menores se apoyan en una figura orientadora respecto al lugar donde se realizan las compras y que está encarnada en un hermano o un niño mayor; igualmente los niños consumen ciertos productos para buscar crear un patrón que los iguale a sus amigos y que se puedan construir relaciones entorno a las similitudes entre ellos.

No hay que olvidar que los niños pasan algunas horas del día expuestos a los comerciales de televisión, cuyo fin es reforzar los estímulos para intervenir en la decisión de compra o adquisición de un producto y no de otro, objetivo que los niños desconocen, ahora bien se suma a lo anterior con la afirmación que establece lo fascinados que se sienten los niños con los comerciales musicales y dinámicos encontramos que los infantes se sienten atraídos por los spots, que se saben sus canciones y que influyen notablemente en el consumo infantil.

En cuanto a la influencia de la escuela, se pudo identificar que aunque desempeña un papel un papel importante dentro de la educación del consumo, en el momento de tomar las decisiones de compra, los niños hicieron pocas alusiones sobre las enseñanzas aprendidas en esos claustros. Siendo así, los resultados apuntan a inferir que la escuela hace orientaciones muy básicas y sus aportes como mecanismo de influencia, no son lo que podrían y deberían ser. Al respecto, Tur y Ramos (2008) hacen alusión a las tareas que debe iniciar la escuela para resaltar su impacto en la educación de consumo en el niño, en aras de ayudar a los infantes a convertirse en consumidores responsables.

\section{REFERENCIAS BIBLIOGRÁFICAS}

Álvarez, A. (2008). "Efectos de la publicidad y los medios de comunicación en el público infantil: desestructurando los valores y forzando la madurez". Nuevas tendencias de la comunicación. Ciclos Complutenses, compilado por Juan Benavides, Elena Fernández, David Alameda y Nuria Villagra, 159-170. Madrid: Editorial Universidad Complutense de Madrid. 
Breé, J. (1995). “Los niños, el consumo y el Marketing”. Madrid: Paidos Ibérica

Carazo, S. (2009). "Comprando a través de los ojos de un niño, el mundo delmarketing infantil". Universidad Latinoamericana de Ciencia y Tecnología Costa Rica. Recuperado 20/03/2014 de <http://www.ulacit.ac.cr/files/careers/93 comprandoatravesdelosojosdeunnioscarazo.pdf $>$ [Consulta 14 de mayo de 2014].

Del Moral Pérez, M. (1999). La publicidad indirecta de los dibujos animados y el consumo infantil de juguetes. Revista comunicar. Grupo comunicar. Recuperado 12/04/2014 de: http://www.redalyc.org/articulo.oa?id=15801335

Lázaro, I. y Mayoral, I. (2005). Infancia, publicidad y consumo. III jornadas sobre derechos de los menores. Universidad Pontificia Comillas. Madrid, España.

Macias, A., Gordillo, L. y Camacho, E. (2012). Hábitos alimentarios de niños en edad escolar y el papel de la educación para la salud, pp 40-43. Chile. Revista chilena de nutrición. Recuperado 3/05/2014 de: http://www.scielo.cl/scielo.php?script=sci_ arttext\&pid $=$ S0717-75182012000300006

Maryam, M. y Mosoomeh, K. (2005). Publicidad sobre Alimentos en los Programas de Televisión Infantiles. Ecology of Food and Nutrition.Recuperado 6/05/2014 de: http://www.bago.com/BagoArg/Biblio/nutriweb224.htm

Mondragón, C. (2013). Dirección estratégica: La influencia de los niños en la compra. Revista de negocios del ITAM. Recuperado 1/05/2014 de: http://direccionestrategica. itam.mx/la-influencia-de-los-ninos-en-la-compra/

Núñez, M. (2013). La influencia de los hermano s en el desarrollo. Recuperado 6/05/2014 de: http://suite101.net/article/la-influencia-de-los-hermanos-en-eldesarrollo-a8802

Otero, M. y Giraldo, W. (2014). La experiencia tecnológica de los niños en su rol de consumidores. Ponencia presentada en el Congress of qualitativeinquiry $\mathrm{X}$, Universidad de Illinois en Urbana-Champaign, 21-24 de mayo.

Pérez, M. (1999). La publicidad indirecta de los dibujos animados y el consumo infantil de juguetes. Revista Comunicar. Recuperado 12/05/2014 en < http://www. redalyc.org/articulo.oa?id=15801335> [Consulta: 12 de mayo de 2014]

Portela, L. (2011). Influencia de los niños en la decisión de compra de los padres. Estudio exploratorio con niños de 6 a 9 años de edad y padres de familia de la ciudad de Santiago de Cali. Tesis de pregrado en Mercadeo y Negocios Internacionales. Cali: Universidad Autónoma de Occidente de Cali.

Restrepo, S. y Maya, M. (2004). La familia y su papel en la formación de los hábitos alimentarios en el escolar. Un acercamiento a la cotidianidad. Red de Revistas Científicas de América Latina, el Caribe, España y Portugal Sistema de Información Científica. Antioquia. Boletín de Antropología universidad de Antioquia. Recuperado 3/05/2014 de: http://www.redalyc.org/articulo.oa?id=55703606.

Rojas, V. (2008). "Influencia de la televisión y videojuegos en el aprendizaje y conducta infanto-juvenil”. Revista chilena de pediatría. Recuperado 07/05/2014 de: http:// www.scielo.cl/scielo.php?pid=S0370-41062008000700012\&script $=$ sci_arttext 
LAURA CATALINA GALLEGO ESQUIVEL/YEHIMI CAROLINA HIGUERA /MARÍA CRISTINA OTERO GÓMEZ /WILSON GIRALDO PÉREZ

Ruiz, M. (1996). Como aprenden a comprar los niños. Acepresa. Recuperado 12/04/2014 de: http://www.aceprensa.com/articles/c-mo-aprenden-a-comprar-losni-os/

Sanday, P. (1981).Female Power and Male Dominance: On the Origins of Sexual Inequality. Cambridge University Press

Schor, J. (2004). Nacidos para comprar. Barcelona, España. Paidós Ibérica S.A

Sedeño, A. (2005). Emoción y hábitos de los niños frente a la televisión. Revista Comunicar. Recuperado 15/02/2014 en <http://www.redalyc.org/articulo. oa?id $=15825055>$

Tur, V. y Ramos, I. (2008). Marketing y niños. Madrid: Esic Editorial

Vygotski, L. (1991). A formação social da mente. São Paulo: MartinsFontes Editora 